the situation. Perhaps he has had a similar task before. He seized the female by the ovipositor and with the help of the other male dragged it out, and walked off quite unconcerned. The female brushed herself and made off in another direction, followed by a grateful male tapping her affectionately with his antennæ.

Dept. of Agriculture, Georgetown, British Guiana, Feb. 17.

\section{Polish on Metals}

Mr. R. C. FRencH has reported on electron diffraction experiments on polished metal surfaces (copper, silver). ${ }^{1}$ The fact that the diffraction rings become more and more diffuse if thesurface is carefully polished is, he concludes, an experimental confirmation of Sir George Beilby's theory, which supposes that by careful polishing a metal surface is covered with a thin amorphous layer of the metal as a supercooled liquid. Mr. H. Raether, who is working in this laboratory with a diffraction apparatus, ${ }^{2}$ has got similar results with polished metals. I do not, however, believe that for the explanation of these results it is necessary to suppose that the size of the crystals which constitute the surface is really altered so very much by the process of polishing. I should like to suggest a simpler explanation. A polycrystalline metal surface which is bombarded with electrons at a grazing angle gives sharp diffraction rings if the surface consists of small lumps which are thin enough to allow the electrons to pass through; this was first shown by Prof. G. P. Thomson. ${ }^{3}$ The lumps play the rôle of the grating, and if by polishing the metal they are levelled, the resolving power of the gratings is gradually diminished (corresponding to the diminished breadth of the gratings).

The sharpness of the diffraction rings proves nothing, therefore, about the real size of the crystals, but gives definite information of the degree of levelling of the surface. Finally, if at the highest degree of polishing the lumps have wholly disappeared, the diffraction would be a specular reflection on the plane surface of the polycrystalline target. In this case refraction would occur and cause a considerable shift of the interference maxima-which would also be broadened-and probably the vanishing of (111) and (002); however, this seerns not to be realised in the present experiments, and requires further investigation.

Institute for Theoretical Physics, F. KIRCHNER. Munich, Feb. 5.

1 NATURE, 129, 169, Jan. 30, 1932.

F. Kircliner, Ann. Phys., 11, 741; 1931

3 G. P. Thomson, Proc. Roy. Soc., A, 128, 650; 1930.

IN connexion with Mr. French's work on polished metal surfaces ${ }^{1}$ and Prof. Kirchner's very interesting suggestion above that diffuse diffraction rings may be due to extreme flatness of the surface rather than to an amorphous state, we should like to mention some results which we have recently obtained.

Surfaces of platinum spluttered on glass have been examined by electron diffraction; they show various patterns, of which one is that given by platinum in its ordinary state. If the platinum is spluttered in oxygen at a voltage not exceeding about 1150 and a pressure of not less than $0.014 \mathrm{~mm}$., the surface is active in promoting the combination of hydrogen and oxygen by catalytic action. Such surfaces show at first diffuse rings very similar to those found by French for polished metals, but after the surface has been used as a catalyst until its activity is lost, these diffuse rings change to the sharp rings characteristic of normal platinum. This change is also produced by heating in vacuo.
It is unlikely that these diffuse rings are due to an extremely flat platinum surface, which one would not expect to be catalytically active, and we find that the change from diffuse to sharp rings is accompanied by an improvement in the specular reflection, suggesting that the surface gets smoother rather than rougher. It seems more likely that the diffuse rings are due to some substance in a state of very fine subdivision, with perhaps inclusions of gas molecules.

$$
\begin{aligned}
& \text { C. A. Murison. } \\
& \text { N. Stuart. } \\
& \text { G. P. Thomson. }
\end{aligned}
$$

Imperial College of Science, S.W.7, March 22.

1 NATURE, 129, 169, Jan. 30, 1932.

\section{Non-polar Auroral Light from the Night Sky in the Tropics}

Is a previous communication from one of us, ${ }^{1}$ it was stated that the brightness of the auroral green line in the northern and southern night skies at Poona (lat. $18^{\circ} 31^{\prime}$ N.) does not show the midnight maximum observed by Lord Rayleigh and by McLennan and his collaborators in temperate latitudes. Further estimates of intensity obtained by exposing Mimosa extreme orthochromatic plates through suitable green and orange filters, and with an aperture of about $45^{\circ}$ towards the zenith, shows definitely that, in general, the brightness of the overhead sky gradually decreases from sunset to a minimum at about midnight, and increases after midnight.

To test whether the result was due to admixture with zodiacal light (it is questionable whether we should consider it as separate from night sky light), simultaneous photographs were taken on a few occasions of the spectrum of the sky with the spectrograph pointed approximately towards the pole star. These confirm that even in the north sky there is a distinct minimum of brightness within an hour of midnight. It may be mentioned that the nights were all perfectly clear. Occasional casual variations, such as have been noted by various observers in Europe and America, also occur.

If the excitation of the green line is directly or indirectly due to ultra-violet light from the sun, one would a priori expect a minimum brightness of the sky some time after midnight depending on the height of the effective layer of atomic oxygen. The maximum brightness observed in temperate latitudes at about this hour is in need of adequate explanation.

K. R. RAMANATHAN.

J. V. KaraNDIKAR.

Meteorological Office,

Ganeshkind Road, Poona 5, Jan. 14.

' [NATURE, 129, 280, Feb. 20, 1932.]

\section{Anomalous Diamagnetism of Bismuth}

BISMUTH when electrically or mechanically colloidalised shows a fall in its diamagnetic susceptibility value. ${ }^{1}$ Doubts were raised by Prof. Bhatnagar as to whether this fall in value could not be due to oxidation. ${ }^{2}$ It was shown by $\mathrm{me}^{3}$ that though a large part of this value was certainly due to oxidation, still there was a decrease in value which could be explained only by attributing it to reduced particle size, as in the case of graphite.

The problem has now been attacked in a different manner. Colloidal bismuth, mechanically prepared and purified by treatment with tartaric acid and alcohol, was investigated in the following way: A small quantity was sealed in vacuo in a small glass bulb. The deflexion was noted in a Curie balance 